\title{
Characterization of heterogeneous vancomycin- intermediate resistance, MIC and accessory gene regulator (agr) dysfunction among clinical bloodstream isolates of staphyloccocus aureus
}

\author{
Yoriko Harigaya ${ }^{1,5}$, Dung $\mathrm{Ngo}^{1}$, Alan J Lesse ${ }^{2}$, Vanthida Huang ${ }^{3}$ and Brian T Tsuji ${ }^{1,4^{*}}$
}

\begin{abstract}
Background: The development of hVISA has been associated with vancomycin clinical failures and is commonly misidentified in clinical microbiology laboratories. Therefore, the objectives of this present study was to improve the reliability of methodologies and criteria for identifying hVISA, evaluate the prevalence of hVISA among clinical bloodstream isolates of $S$. aureus and determine if there exists a relationship between accessory gene regulator (agr) dysfunction and the hVISA phenotype.

Methods: The presence of hVISA in 220 clinical S. aureus isolates (121 MSSA, 99 MRSA) from bloodstream infections was examined by CLSI broth microdilution, Macro \& Standard Etest. Isolates which were classified as hVISA by Macro Etest, were additionally evaluated using a modified PAP-AUC method using a modified starting inoculum of $10^{10} \mathrm{CFU} / \mathrm{mL}$, and growth on brain heart infusion agar with $4 \mathrm{mg} / \mathrm{L}$ vancomycin (BHIV4) at $10^{8}$ and $10^{10} \mathrm{CFU} / \mathrm{mL}$, and agr function was assessed by delta-hemolysin production.

Results: Broth microdilution $\mathrm{MIC}_{50 / 90}$ of S.aureus and hVISA was 1.0/2.0 and 1.5/2.0 mg/L ( $\left.p=0.02\right)$, respectively. Macro Etest identified 12 (5.5\%) hVISA isolates; higher among MRSA (9.1\%) versus MSSA (2.5\%) $(p=0.03)$. The mean modified PAP-AUC ratios ( $>0.8)$ of 7 MRSA strains and 3 MSSA strains were significantly different $(p=0.001)$. $58 \%$ of hVISA strains were found to be agr dysfunctional when $21 \%$ of MRSA strains were agr dysfunctional. hVISA was detected among S. aureus bloodstream isolates, which were classified as susceptible among clinical microbiology laboratories.

Conclusions: Evaluating the correlation between Etest MICs and modified PAP-AUC ratio values will add further improvement of discriminating hVISA, and agr dysfunction may be predictive of strains which display a greater predilection to display the hVISA phenotype.
\end{abstract}

\section{Background}

Heteroresistant vancomycin-intermediate Staphylococcus aureus (hVISA) has been detected in clinical S. aureus strains throughout the world [1-3] and has been associated with vancomycin treatment failure for $S$. aureus [4]. The prevalence of hVISA has been reported ranging from 0 to $74 \%[2,5]$. The large variance of the hVISA

\footnotetext{
* Correspondence: btsuji@buffalo.edu

'Laboratory for Antimicrobial Pharmacodynamics, School of Pharmacy and Pharmaceutical Sciences and The New York State Center of Excellence in Bioinformatics \& Life Sciences, University at Buffalo, State University of New York, New York, USA

Full list of author information is available at the end of the article
}

prevalence is due to the lack of a prevalence study. On top of that, standardized methodologies and criteria to detect hVISA in the clinical microbiology laboratory are not established. The prevalence study with high quality cost effective methodologies are necessary to be conducted with uniformly to identify the true hVISA prevalence. Owing to the unstable characteristics of vancomycin heteroresistant phenotype, establishment of standardized methods to detect hVISA has been challenging [5].

hVISA was first characterized in 1997 [6]. hVISA is defined as isolates which are classified by the Clinical 
and Laboratory Standard Institute (CLSI) as having a vancomycin broth microdilution minimum inhibitory concentration (MIC) in the susceptible range where a notable subpopulation of cells (approximately one organism in every $10^{5}-10^{6}$ or more) with MICs over the susceptibility breakpoint [6]. A number of current methodologies for detection of hVISA exist such as the Etest Macromethod, Etest Glycopeptide Resistance Detection (Etest GRD: Standard Etest) method and the population analysis profile-area under the curve (PAP-AUC) method [7]. Multiple studies demonstrated high sensitivity, high specificity, consistent and low variation between laboratories in detecting hVISA by the Macro Etest Method [8-10]. Although PAP-AUC is labor intensive, it has long been considered the gold standard for hVISA detection [2]. Furthermore, as it relates to genetic factors which can be markers for hVISA, no single resistance gene has been correlated with hVISA development [11,12]; however, it has been demonstrated that the expression of hVISA phenotype is well associated with dysfunction in the quorum sensing cluster of genes, the accessory gene regulator group (agr), controlling colonization and virulence, as well as a number of other regulators [13].

In this study, the objectives were to: 1) determine the prevalence of hVISA in clinical S. aureus bloodstream isolates utilizing Macro Etest, Standard Etest, modified PAP-AUC method and Brain Heart Infusion Agar containing vancomycin $4 \mathrm{mg} / \mathrm{L}$ (BHIAV4) method. 2) determine the relationship between hVISA, vancomycin MIC, and $a g r$ dysfunction among clinical S. aureus bloodstream isolates for the purpose of establishing the standardized method to facilitate the hVISA detection in the clinical setting.

\section{Methods}

\section{Bacterial Isolates}

220 clinical S. aureus isolates obtained from the blood of infected patients at the Buffalo Veterans Affairs Health System of Western New York from 2003 to 2005 were analyzed. Isolates were obtained from 220 different patients. All studies were conducted in accordance with the Institutional review board at the University at Buffalo and the VA Health System. Control MSSA strain $S$. aureus ATCC29213 and a standard hVISA strain Mu3 (ATCC700698) were utilized.

\section{Antimicrobial agents, Media, and MIC Determination}

Vancomycin powder (Sigma Chemical Co, St. Louis, MO) was obtained commercially. Stock solutions were made according to manufacturer's directions and stored at $4^{\circ} \mathrm{C}$. Etest strips of vancomycin (AB Biodisk, Solna, Sweden) were utilized for the initial screening of hVISA. All susceptibility testing used Mueller-Hinton Broth
(Difco Laboratories, Detroit, MI) supplemented with calcium $(25 \mathrm{mg} / \mathrm{L})$ and magnesium $(12.5 \mathrm{mg} / \mathrm{L})$. BrainHeart Infusion agar (Difco Laboratories, Detroit, MI) was utilized for all analysis. Trypticase soy agar with 5\% sheep blood agar (TSA II, Becton-Dickinson Diagnostics, Sparks, MD) was utilized for a bacteria growth medium and delta-hemolysin test. CLSI Broth microdilution MICs were determined for 220 clinical isolates and a control MSSA strain S. aureus ATCC29213 and a standard hVISA strain Mu3 in triplicate accordance with the Clinical and Laboratory Standards Institute (formerly the National Committee for Clinical Laboratory Standards) guidelines [14].

\section{Macro and Standard Etest}

The detection of hVISA among 220 isolates was completed by using the Etest Macro Method as a preliminary screen as previously described $[7,15]$. Overnight growth cultures of the 220 clinical isolates and a control MSSA strain S. aureus ATCC29213 and a standard hVISA strain Mu3 were suspended in saline solution. The suspensions were adjusted to 2.0 McFarland, swabbed on the BHI plates and dried at room temperature, and Etest strips were placed on the plates. After 48 $\mathrm{h}$ incubation at $35^{\circ} \mathrm{C}$, the intersections of the elliptical inhibition zone and the subpopulation growth in the inhibition zone were recorded. The criterion for Macro Etest for detecting hVISA was the appearance of one or more subpopulation colonies in $\mathrm{MIC} \geq 4 \mathrm{mg} / \mathrm{L}$ refers to the recent study results performed by Maor [16]. Additionally, Standard Etest was performed at a 0.5 McFarland using MHA that was also performed in the similar manner and interpreted according to CLSI breakpoints.

\section{Modified PAP-AUC}

Isolates which displayed hVISA profile by the Macro Etest Method were further analyzed using modified PAP-AUC method according to the standard PAP-AUC methodology as previously described [7]. Briefly, overnight growth culture of hVISA clinical isolates and a control isolates S. aureus ATCC29213 and a standard hVISA strain Mu3 were suspended in saline solution. The suspensions were adjusted to the modified starting inoculum $10^{10} \mathrm{CFU} / \mathrm{mL}$. Cultures were serially diluted from 0 to $10^{-6}$, and $10 \mu \mathrm{L}$ of each dilution was plated in quadruplicate on BHI containing vancomycin in the following concentrations: $0.5,1,2,4,6,8$ and $16 \mathrm{mg} / \mathrm{L}$. Colonies were enumerated after $48 \mathrm{~h}$ incubation at $35^{\circ}$ C. Bacterial colony counts $\left(\log _{10} \mathrm{CFU} / \mathrm{mL}\right)$ were plotted against the vancomycin concentration $(0$ to $4 \mathrm{mg} / \mathrm{L}$ ) using SigmaPlot 9.0. The area under the curve (AUC) was calculated for each isolate and divided by the AUC value of the reference strain Mu3. The criteria for identifying hVISA were either the modified PAP-AUC ratio 
of $>0.9$ or $>0.8$. Additionally, modified PAP-AUC ratios were plotted against Macro Etest MICs, which were evaluated for the relation between two variables using the linear regression equation and the correlation coefficient (r).

\section{BHIAV4}

To evaluate the inoculum effect of hVISA strains detected by the Macro Etest Method, a modified procedure involving Brain Heart Infusion agar contain vancomycin $4 \mathrm{mg} / \mathrm{L}$ BHIAV4 method was performed as described previously [6]. Briefly, overnight growth culture of hVISA clinical isolates and a control isolates $S$. aureus ATCC29213 and a standard hVISA strain Mu3 were suspended in saline solution. The suspensions were adjusted to the modified inoculum of $10^{8}$ and an additional higher inoculum of $10^{10} \mathrm{CFU} / \mathrm{mL}$. Each sample was plated in quadruplicate on $\mathrm{BHI}$ agar containing vancomycin $4 \mathrm{mg} / \mathrm{mL}$. Colonies were enumerated after $48 \mathrm{~h}$ incubation at $35^{\circ} \mathrm{C}$. Growth of 1 or more colonies indicate a positive result.

\section{Delta-hemolysin}

To evaluate the relationship between agr dysfunction and hVISA, delta-hemolysin expression was determined for hVISA isolates detected by Etest according to the study previously described [13]. Briefly, overnight growth culture of hVISA clinical isolates and S. aureus RN4220 were suspended in saline solution. The suspensions were adjusted to $0.5 \mathrm{McF}$ arland standards and were streaked vertically near RN4220 on TSA II. After incubating plates at $35^{\circ} \mathrm{C}$ for $24 \mathrm{~h}$, the expression of delta-hemolysin was evaluated.

\section{Statistical analysis}

The relation between MICs $(\mathrm{mg} / \mathrm{L})$ values and modified PAP-AUC ratios was evaluated using the linear regression analysis. The correlation coefficient (r) was calculated to display the correlation between the two variables. The relationships between the independent and dependent variables were analyzed using MannWhitney $U$ test and chi-square test. P-value of $<0.05$ was considered statistically significant. The number of false positive, false negative, the percentage of sensitivity and specificity were calculated for each method.

\section{Results}

The proportion of methicillin susceptible $S$. aureus (MSSA) and MRSA isolates in tested S. aureus isolates were 55 and $45 \%$, respectively (Table 1 ). The CLSI broth microdilution MIC range for $S$. aureus was 0.25 to $2 \mathrm{mg} / \mathrm{L}$, and for hVISA was 1 to $2 \mathrm{mg} / \mathrm{L}$. The distribution of MICs $0.25,0.5,1$ and $2 \mathrm{mg} / \mathrm{L}$ measured by the broth microdilution method were $6(2.7 \%), 21$
(9.5\%), 159 (72.3\%) and 34 (15.5\%), respectively (Figure $1) . \mathrm{MIC}_{50}$ of S. aureus $(\mathrm{n}=220)$, MSSA $(\mathrm{n}=121)$, MRSA ( $\mathrm{n}=99$ ) and hVISA (determined by Macro Etest, $\mathrm{n}=12$ ) were $1,1,1$ and $1.5 \mathrm{mg} / \mathrm{L}$, respectively. $\mathrm{MIC}_{50 /}$ 90 of hVISA analyzed by broth microdilution, Standard Etest, and Macro Etest were 1.5/2, 4/4, and 6/8 mg/L, respectively.

All tested S. aureus isolates displayed CLSI broth microdilution MIC values in the susceptible range, however, the distribution of MICs was significantly different between the total clinical $S$. aureus and hVISA strains $(p=0.02)$. Standard Etest applying the CLSI breakpoints identified 13 clinical S. aureus strains as hVISA, and Macro Etest classified 1 out of those 13 hVISA strains as a vancomcyin susceptible $S$. aureus (VSSA) which also displayed the low modified PAPAUC ratio (0.54), as shown in PAP profiles in Figure 2. The criterion for Macro Etest for detecting hVISA was the appearance of one or more subpopulation colonies in $\mathrm{MIC} \geq 4 \mathrm{mg} / \mathrm{L}$. hVISA prevalence was higher in MRSA (9.1\%) compared to MSSA $(2.5 \%)(p$ $=0.03$ ) (Table 2). The Etest MIC results displayed high reproducibility (SD < 10\%).

With ten hVISA strains screened by Macro Etest and confirmed by modified PAP-AUC with the ratio cutoff value $>0.8$, the mean modified PAP-AUC ratio $( \pm$ SD) was $(0.91 \pm 0.06)$. Additionally, the mean modified PAPAUC ratio $( \pm S D)$ of seven hVISA isolates with MRSA phenotype was $(0.94 \pm 0.04)$, which was higher compared to the mean ratio of three hVISA isolates with MSSA phenotype $(0.85 \pm 0.02)(p=0.001)$. With a higher endpoint criterion modified PAP-AUC ratio > 0.9 , five hVISA strains were misclassified as susceptible. The percentage of the sensitivity modified PAP-AUC ratio $>0.8$ and ratio $>0.9$ in discriminating hVISA were $83.3 \%$ and $58.3 \%$, respectively, when the sensitivity of broth microdilution, Standard Etest, BHIAV4 with lower inoculum $\left(10^{8} \mathrm{CUF} / \mathrm{mL}\right)$ and BHIAV4 with higher inoculum $\left(10^{10} \mathrm{CFU} / \mathrm{mL}\right)$ were $0 \%, 100 \%, 8.3 \%$ and $100 \%$, respectively.

Macro Etest MICs were plotted against modified PAPAUC ratios (Figure 3). The regression line for the data of thirteen isolates identified as hVISA by Standard Etest is displaying the strong relationship between Macro Etest MIC shift and modified PAP-AUC ratio $\left(\mathrm{r}^{2}\right.$ $=0.78)$. Standard Etest also displayed the relationship between MIC values and modified PAP-AUC ratio $\left(\mathrm{r}^{2}=\right.$ 0.24). In contrast, the CLSI broth microdilusiton MIC values did not have clear correlation with modified PAP-AUC ratios $\left(r^{2}=0.026\right)$. According to the linear regression equation for these two variables, Macro Etest MICs and modified PAP-AUC ratio, the estimated modified PAP-AUC ratio at MIC 4 and $6 \mathrm{mg} / \mathrm{L}$ were 0.62 and 0.85 , respectively. 
Table 1 Summary of the prevalence of MRSA and hVISA in 220 S.aureus bloodstream isolates

\begin{tabular}{|c|c|c|c|c|}
\hline & $\begin{array}{l}\text { S. aureus } \\
220\end{array}$ & $\begin{array}{c}\text { MSSA } \\
55 \%(121 / 220)\end{array}$ & $\begin{array}{c}\text { MRSA } \\
45 \%(99 / 220)\end{array}$ & $\begin{array}{c}\text { hVISA } \\
5.5 \%(12 / 220)\end{array}$ \\
\hline MIC50/90 & $1 / 2$ & $1 / 2(p=0.51)^{a}$ & $1 / 2(p=0.46)^{a}$ & $1.5 / 2(p=0.03)^{a}$ \\
\hline agr-dysfunction & $15 \%(33 / 220)$ & $10 \%(12 / 121)$ & $21 \%(21 / 99)(p=0.02)^{b}$ & $58 \%(7 / 12)(p<0.0005)^{c}$ \\
\hline
\end{tabular}

$\mathrm{MIC}_{50 / 90}$ determined by CLSI broth microdilution and the prevalence of agr-dysfunctional

${ }^{a}$ MIC distribution of total clinical S.aureus versus MIC distribution of each phenotype (Mann-Whitney U)

${ }^{b}$ Prevalence of agr dysfunction in MRSA isolates versus MSSA isolates (Chi-square test)

c Prevalence of agr dysfunction in hVISA isolates versus VSSA isolates (Chi-square test)

The percentages of agr dysfunctional phenotype in total tested S.aureus, MSSA, MRSA and hVISA were $15 \%, 10 \%, 21 \%$ and $58 \%$ (Table 1). The prevalence of agr dysfunctional phenotype was significantly higher in hVISA compared with VSSA $(p=0.02)$. Additionally, the prevalence of $a g r$ dysfunctional phenotype was higher in MRSA compared with MSSA $(p=0.02)$. BHIAV4 method analysis using lower inoculum $\left(10^{8}\right.$ $\mathrm{CFU} / \mathrm{mL}$ ) misclassified 11 out of $12 \mathrm{hVISA}$ strains as VSSA. On the contrary, BHIAV4 analysis with higher inoculum $\left(10^{10} \mathrm{CFU} / \mathrm{mL}\right)$ identified all 12 hVISA strains as hVISA.

\section{Discussion}

Due to the heteroresistant nature of vancomycin intermediate resistance, a number of clinical $S$. aureus may exhibit resistant subpopulations not expressed by traditional methods of MIC detection. Therefore, it is necessary to investigate a variety of methods to increase sensitivity of hVISA detection in clinical laboratories. A recent multicenter, multi-national comparison by Wootton et. al. determined that the Macro Etest method displayed high sensitivity (85.9\%) in detection of hVISA which is rationale of using this method as an initial screen for strains which display hVISA [9]. In the current study we utilized this methodology to determine that the hVISA phenotype was nearly four times higher among MRSA vs. MSSA. Quantitatively, these results were also confirmed by population analysis profiling methods whereby MRSA displayed the higher modified PAP-AUC ratios compared to hVISA MSSA.

This perhaps is intuitive due to vancomycin being commonly utilized for MRSA; however, hVISA was also detected among MSSA, which is in agreement with the recent findings of a significant increase in vancomycin MICs among MSSA clinical isolates by Wang [17]. These findings may suggest that the use of aggressive vancomycin regimens, in MRSA infections are important, so that the development hVISA phenotype which is driven by suboptimal vancomycin therapeutic exposure can be avoided. Certainly, alternative treatment should be used for MSSA infections in lieu of vancomycin treatment.

The BHIAV4 method described by Hiramatsu [6] has been evaluated in a number of studies. One concern with this methodology is that, the heteroresistant subpopulations arise in very low frequency, which results inability of the BHIAV4 method to detect heteroresistant phenotypes when the inoculum is low. To study the impact of inoculums on detection and mutation frequency, we utilized a high inoculum of $S$. aureus $\left(10^{10}\right.$ $\mathrm{CFU} / \mathrm{ml}$ ) which is often observed in the severe S. aureus
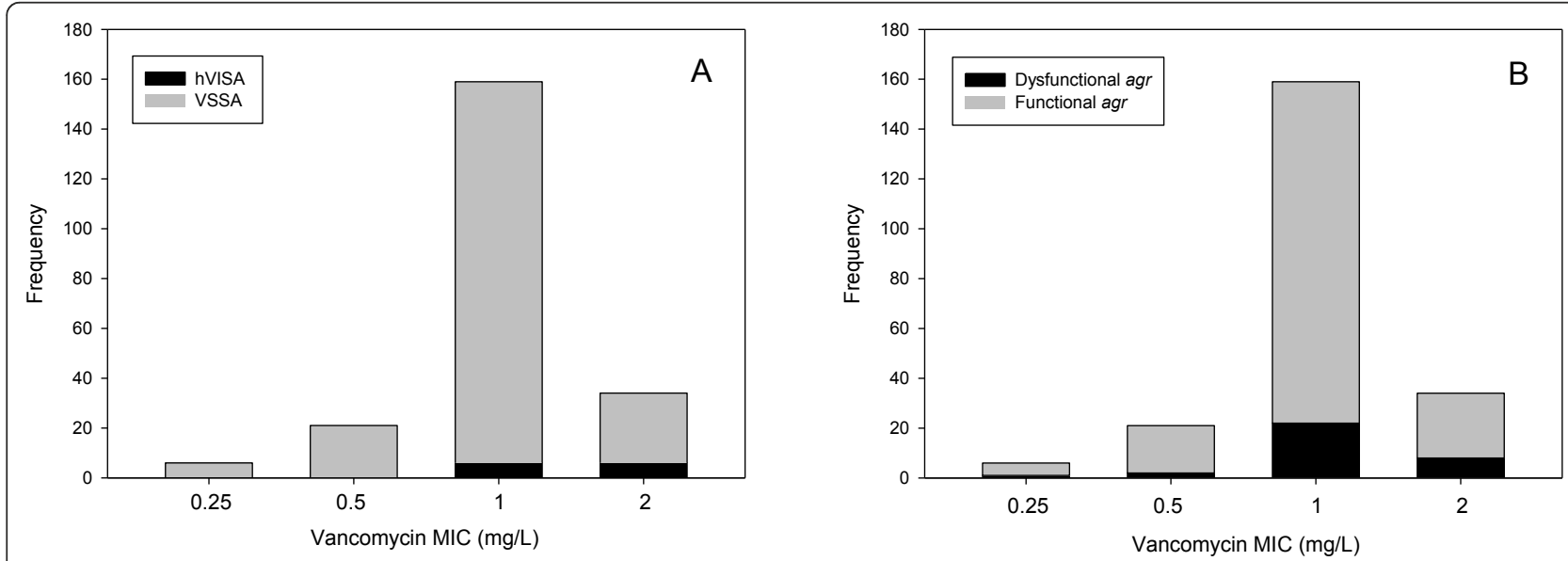

Figure 1 MIC distribution of 220 S. aureus bloodstream isolates in relation to frequency of isolates that were hVISA (A) or agrdysfunction (B). 


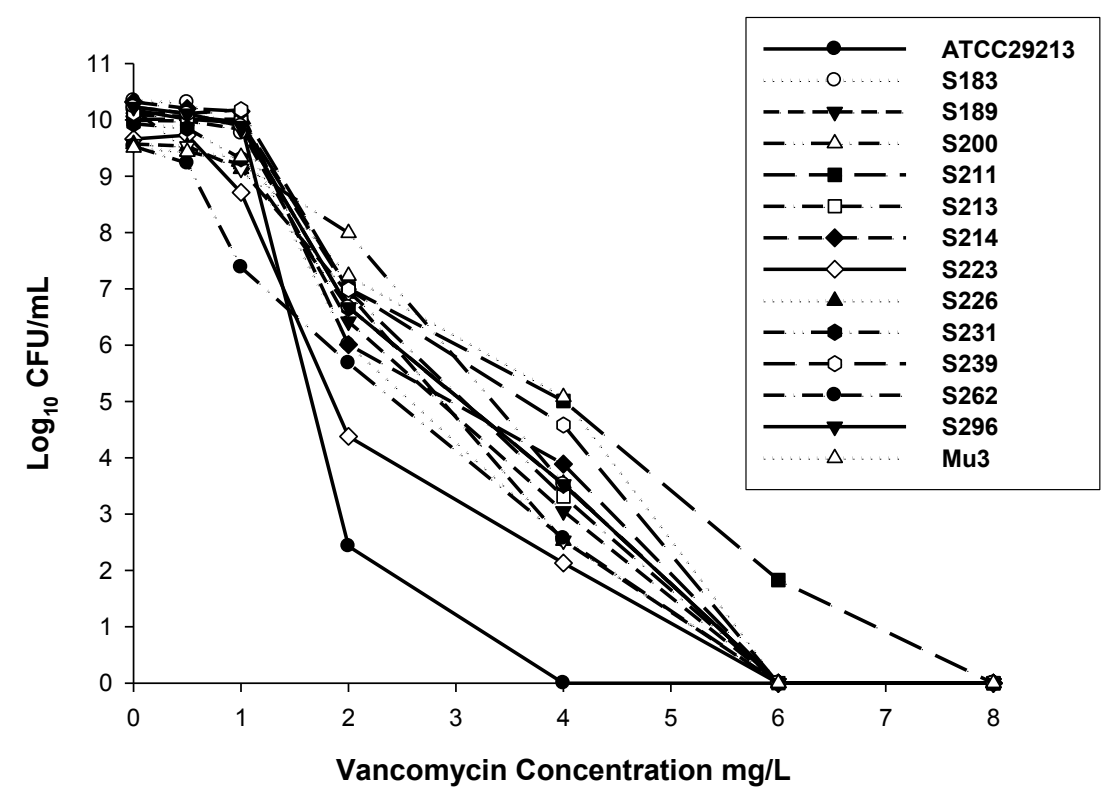

Figure 2 Population analysis profiles of twelve hVISA clinical isolates, a control isolates S. aureus ATCC29213 and a standard hVISA strain Mu3.

infection including infective endocarditis. As a result, the inoculum level displayed the significant impact on the sensitivity: the modified BHIAV4 method using the higher inoculum $10^{10} \mathrm{CUF} / \mathrm{mL} 100 \%$ isolates additional hVISA correctly. Furthermore, at high bacterial density, stationary-phase growth foster biofilm formation via quorum-sensing mechanisms involving agr in S. aureus [13]. Coupled with the finding that proportion of $a g r$ dysfunctional strains were nearly five times higher among hVISA vs. VSSA, these findings may provide additional potential mechanistic insights into the development vancomycin resistance. Furthermore, agr dysfunctional $S$. aureus strains tend to display low activity in metabolic pathway which is growth phase dependant. Therefore, in the context of high bacterial density, it has been hypothesized that stationary phase growth, defective autolysis profiles, biofilim production and the production of thicker cell walls, may be facilitated by quorum sensing mechanisms [11-13]. Finally, from a clinical microbiology standpoint, the evaluation of delta-

Table 2 Summary of the shift in MIC and PAP-AUC ratio of 13 hVISA isolates screened by Standard Etest and a control S. aureus ATCC29213.

\begin{tabular}{|c|c|c|c|c|c|c|c|}
\hline ID & Organism & BMD $^{1}$ & Standard Etest & Macro Etest & PAP-AUC Ratio & hVISA $^{2}$ & agr-function \\
\hline S183 & MSSA & 2 & 3 & 6 & 0.83 & Y & Positive \\
\hline S189 & MSSA & 2 & 4 & 6 & 0.87 & Y & Negative \\
\hline S200 & MRSA & 1 & 3 & 6 & 0.95 & Y & Negative \\
\hline S211 & MRSA & 1 & 4 & 8 & 1.12 & Y & Positive \\
\hline S213 & MRSA & 1 & 4 & 8 & 0.91 & Y & Positive \\
\hline S214 & MRSA & 2 & 6 & 6 & 0.91 & Y & Positive \\
\hline S223 & MRSA & 2 & 4 & 6 & 0.7 & Y & Positive \\
\hline S226 & MSSA & 2 & 3 & 6 & 0.84 & Y & Negative \\
\hline S231 & MRSA & 1 & 4 & 6 & 0.9 & Y & Negative \\
\hline S239 & MRSA & 2 & 4 & 8 & 0.99 & Y & Negative \\
\hline S262 & MRSA & 1 & 3 & 4 & 0.75 & Y & Positive \\
\hline S285 & MRSA & 1 & 3 & 3 & 0.54 & N & Negative \\
\hline S296 & MRSA & 1 & 3 & 6 & 0.91 & Y & Negative \\
\hline ATCC29213 & MSSA & 1 & 2 & 3 & 0.53 & N & Positive \\
\hline
\end{tabular}

'BMD: Broth Microdilution

${ }^{2}$ Identified by Macro Etest 


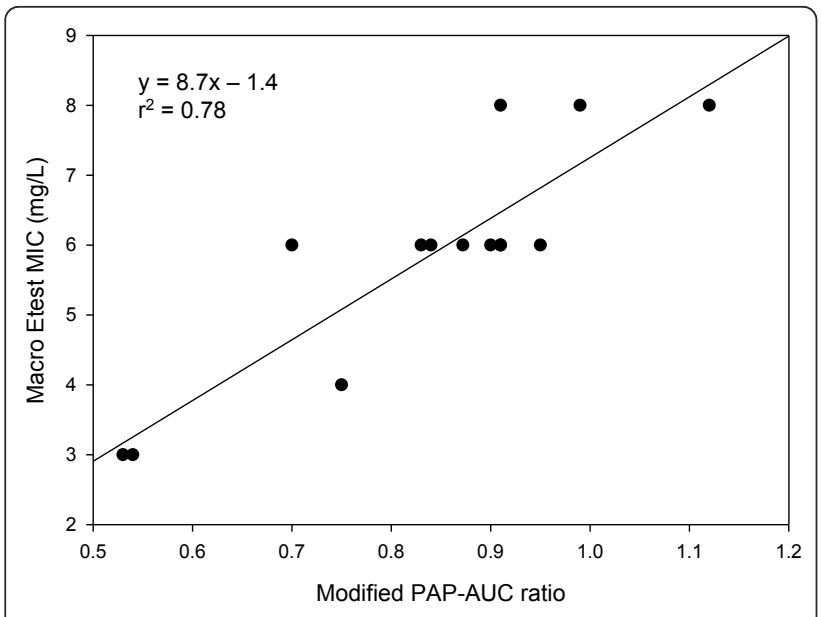

Figure 3 Scatter plot displaying the correlation between Macro Etest MICs and modified PAP-AUC ratio of thirteen S. aureus clinical isolates identified as hVISA by Standard Etest and control isolate ATCC29213.

hemolysin production may be of potential utility to detect staphylococci which display an increase proclivity to develop heterogeneous resistance.

This study had potential limitations. First, we acknowledge the lack of geographically diverse sites for S. aureus, as isolates were from a single institution and limited to nosocomial bacteremia in a defined geographic area. Additional multicenter studies including both nosocomial and community-onset bloodstream infections are necessary to define the true prevalence of hVISA. Second, we utilized PAP using a high initial inoculum of $10^{10} \mathrm{CFU} / \mathrm{mL}$ to confirm all hVISA isolates determined by the Etest Macro Method. Although, this method may decrease specificity, our VSSA control strain, ATCC 29213 did not display any vancomycin intermediate populations. Third, although we utilized delta-hemolysin expression as a surrogate marker measure of agr function, more quantitative measure of RNAIII expression may be necessary to further define the relationship $a g r$ and heteroresistance. Overall, the prevalence of hVISA phenotype in 220 clinical S. aureus isolates was observed to be 5.5\% in S. aureus and 9.1\% in MRSA strains using the Etest Macro Method, which was well correlated with modified PAP-AUC ratio values. agr dysfunction may add further utility to hVISA detection in the clinical microbiology setting.

\section{Conclusion}

hVISA was detected among S. aureus bloodstream isolates, which were classified as susceptible among clinical microbiology laboratories. Evaluating the correlation between Etest MICs and modified PAP-AUC ratio values will add further improvement of discriminating
hVISA, and agr dysfunction may be predictive of strains which display a greater predilection to display the hVISA phenotype.

\section{Acknowledgements and funding}

We would like to thank Michael Ma for excellent technical assistance. This study was funded by the University at Buffalo, State University of New York, Interdisciplinary Research Fund.

\section{Author details}

'Laboratory for Antimicrobial Pharmacodynamics, School of Pharmacy and Pharmaceutical Sciences and The New York State Center of Excellence in Bioinformatics \& Life Sciences, University at Buffalo, State University of New York, New York, USA. ${ }^{2}$ School of Medicine and Biomedical Sciences, University at Buffalo, State University of New York, New York, USA. ${ }^{3}$ Mercer University, College of Pharmacy, Atlanta, Georgia, USA. ${ }^{4}$ Roswell Park Cancer Institute Departments of Medicine, Buffalo, New York, USA. ${ }^{5}$ Food and Drug Administration, Center for Drug Evaluation and Research, Silver Spring, Maryland, USA.

\section{Authors' contributions}

$\mathrm{YH}$ was responsible for overall study design, participated in the experimental work, conducted an extensive literature review, and wrote the manuscript. DN carried out the experimental work and participated in study design. VH participated in the study design and contributed to writing of the manuscript. AJL contributed ideas and characterization of strains. BTT was responsible for overall study design, conducted an extensive literature review, and wrote the manuscript. All authors have read and approved the final manuscript.

\section{Competing interests}

The authors declare that they have no competing interests.

Received: 17 November 2010 Accepted: 25 October 2011

Published: 25 October 2011

\section{References}

1. Howe RA, Monk A, Wootton M, Walsh TR, Enright MC: Vancomycin susceptibility within methicillin-resistant Staphylococcus aureus lineages. Emerg Infect Dis 2004, 10:855-857.

2. Walsh TR, Howe RA: The prevalence and mechanisms of vancomycin resistance in Staphylococcus aureus. Annu Rev Microbiol 2002, 56:657-675.

3. Maor Y, Hagin M, Belausov N, Keller N, Ben-David D, Rahav G: Clinical features of heteroresistant vancomycin-intermediate Staphylococcus aureus bacteremia versus those of methicillin-resistant $S$. aureus bacteremia. J Infect Dis 2009, 199:619-624.

4. Appelbaum PC: Reduced glycopeptide susceptibility in methicillinresistant Staphylococcus aureus (MRSA). Int J Antimicrob Agents 2007, 30:398-408.

5. Liu C, Chambers HF: Staphylococcus aureus with heterogeneous resistance to vancomycin: epidemiology, clinical significance, and critical assessment of diagnostic methods. Antimicrob Agents Chemother 2003, 47:3040-3045.

6. Hiramatsu K, Aritaka N, Hanaki H, Kawasaki S, Hosoda Y, Hori S, Fukuchi Y, Kobayashi I: Dissemination in Japanese hospitals of strains of Staphylococcus aureus heterogeneously resistant to vancomycin. Lancet 1997, 350:1670-1673.

7. Wootton M, Howe RA, Hillman R, Walsh TR, Bennett PM, MacGowan AP: A modified population analysis profile (PAP) method to detect heteroresistance to vancomycin in Staphylococcus aureus in a UK hospital. J Antimicrob Chemother 2001, 47:399-403.

8. Voss A, Mouton JW, van Elzakker EP, Hendrix RG, Goessens W, Kluytmans JA, Krabbe PF, de Neeling HJ, Sloos JH, Oztoprak N, et al: A multi-center blinded study on the efficiency of phenotypic screening methods to detect glycopeptide intermediately susceptible Staphylococcus aureus (GISA) and heterogeneous GISA (h-GISA). Ann Clin Microbiol Antimicrob 2007, 6:9.

9. Wootton M, MacGowan AP, Walsh TR, Howe RA: A multicenter study evaluating the current strategies for isolating Staphylococcus aureus 
strains with reduced susceptibility to glycopeptides. J Clin Microbiol 2007, 45:329-332.

10. Yusof A, Engelhardt A, Karlsson A, Bylund L, Vidh P, Mills K, Wootton M, Walsh TR: Evaluation of a new Etest vancomycin-teicoplanin strip for detection of glycopeptide-intermediate Staphylococcus aureus (GISA), in particular, heterogeneous GISA. J Clin Microbiol 2008, 46:3042-3047.

11. Howden BP, Smith DJ, Mansell A, Johnson PD, Ward PB, Stinear TP, Davies JK: Different bacterial gene expression patterns and attenuated host immune responses are associated with the evolution of low-level vancomycin resistance during persistent methicillin-resistant Staphylococcus aureus bacteraemia. BMC Microbiol 2008, 8:39.

12. Wootton M, Avison MB, Bennett PM, Howe RA, MacGowan AP, Walsh TR: Genetic analysis of 17 genes in Staphylococcus aureus with reduced susceptibility to vancomycin (VISA) and heteroVISA. J Antimicrob Chemother 2004, 53:406-407.

13. Sakoulas G, Eliopoulos GM, Moellering RC Jr, Wennersten C, Venkataraman L, Novick RP, Gold HS: Accessory gene regulator (agr) locus in geographically diverse Staphylococcus aureus isolates with reduced susceptibility to vancomycin. Antimicrob Agents Chemother 2002, 46:1492-1502.

14. CLSI, Institute CaLS: Performance standards for antimicrobial susceptibility testing, 15th informational supplement M100-S15. Book Performance standards for antimicrobial susceptibility testing, 15th informational supplement M100-S15 City: CLSI; 2005, (Editor ed.^eds.)

15. Walsh TR, Bolmstrom A, Qwarnstrom A, Ho P, Wootton M, Howe RA, MacGowan AP, Diekema D: Evaluation of current methods for detection of staphylococci with reduced susceptibility to glycopeptides. Journal of clinical microbiology 2001, 39:2439-2444.

16. Maor Y, Rahav G, Belausov N, Ben-David D, Smollan G, Keller N: Prevalence and characteristics of heteroresistant vancomycin-intermediate Staphylococcus aureus bacteremia in a tertiary care center. J Clin Microbiol 2007, 45:1511-1514

17. Wang G, Hindler JF, Ward KW, Bruckner DA: Increased Vancomycin MICS for Staphylococcus aureus Clinical Isolates from a University Hospital during a 5-Year Period. J Clin Microbiol 2006, 44:3883-3886.

\section{Pre-publication history}

The pre-publication history for this paper can be accessed here: http://www.biomedcentral.com/1471-2334/11/287/prepub

doi:10.1186/1471-2334-11-287

Cite this article as: Harigaya et al:: Characterization of heterogeneous vancomycin-intermediate resistance, MIC and accessory gene regulator (agr) dysfunction among clinical bloodstream isolates of staphyloccocus aureus. BMC Infectious Diseases 2011 11:287.

\section{Submit your next manuscript to BioMed Central and take full advantage of:}

- Convenient online submission

- Thorough peer review

- No space constraints or color figure charges

- Immediate publication on acceptance

- Inclusion in PubMed, CAS, Scopus and Google Scholar

- Research which is freely available for redistribution

Submit your manuscript at www.biomedcentral.com/submit
Biomed Central 